This is a self-archived version of an original article. This version may differ from the original in pagination and typographic details.

Author(s): Mäntysaari, Heikki

Title: Exclusive Vector Meson Production at the EIC

Year: 2020

Version: Accepted version (Final draft)

Copyright: (c) World Scientific, 2020

Rights: In Copyright

Rights url: http://rightsstatements.org/page/InC/1.0/?language=en

Please cite the original version:

Mäntysaari, H. (2020). Exclusive Vector Meson Production at the EIC. In A. Prokudin, Y. Hatta, Y. Kovchegov, \& C. Marquet (Eds.), Probing Nucleons and Nuclei in High Energy Collisions :

Proceedings of INT Program INT-18-3 (pp. 262-266). World Scientific.

https://doi.org/10.1142/9789811214950_0053 


\title{
Exclusive vector meson production at the EIC
}

\author{
Heikki Mäntysaari* \\ Department of Physics, University of Jyväskylä, P.O. Box 35, 40014 University of Jyväskylä, \\ Finland, and \\ Helsinki Institute of Physics, P.O. Box 64, 00014 University of Helsinki, Finland \\ *E-mail: heikki.mantysaari@jyu.fi
}

\begin{abstract}
We discuss how vector meson production at the future Electron Ion Collider can be used to probe non-linear dynamics in heavy nuclei. Additionally, the potential to study the
\end{abstract} evolution of proton and nuclear geometries with event-by-event fluctuations is illustrated.

\section{Introduction}

Exclusive vector meson production is a very powerful tool to probe the internal structure of protons and nuclei. In these processes no color string between the target and the produced particle can exist (as it would break and produce many particles). Thus, there can not be net color transfer to the target, which in perturbative QCD requires at least two gluons to be exchanged. Consequently, the cross section is approximatively proportional to the squared gluon distribution ${ }^{1}$.

Good sensitivity on the small- $x$ gluonic structure makes exclusive processes potentially very powerful in studies of non-linear saturation effects. As the gluon densities rise towards small $x$ due to the enhanced radiation of soft gluons in QCD, at some point it is expected that one reaches the limit where non linear effects tame this growth. As a result, anew state of matter where color fields are as strong as allowed in the nature is formed. An effective field theory describing QCD in this regime is known as the Color Glass Condensate ${ }^{2}$.

Additionally, in exclusive process the total momentum transfer can be measured by reconstructing the momentum of the produced particle and that of the outgoing lepton. As the transverse momentum transfer is the Fourier conjugate to the impact parameter, these processes at high energies provide access to the spatial distribution of small Bjorken- $x$ gluons in the target wave function, as well as to the event-byevent geometry fluctuations as discussed below.

\section{Proton structure}

At high energies, the cross section for the exclusive production of the vector meson $V$ can be written as ${ }^{5}$

$$
\mathcal{A}_{T, L}^{\gamma^{*} p \rightarrow V p}=i \int \mathrm{d}^{2} \mathbf{r} \int \mathrm{d}^{2} \mathbf{b} \int \frac{\mathrm{d} z}{4 \pi}\left(\Psi^{*} \Psi_{V}\right)_{T, L}\left(Q^{2}, \mathbf{r}, z\right) e^{-i[\mathbf{b}-(1-z) \mathbf{r}] \cdot \Delta} 2 N\left(\mathbf{r}, \mathbf{b}, x_{\mathbb{P}}\right),
$$

where $\Psi^{*} \Psi_{V}$ is the overlap between the virtual photon wave function (computed from QED) and the vector meson wave function (modelled), and the dipole ampli- 


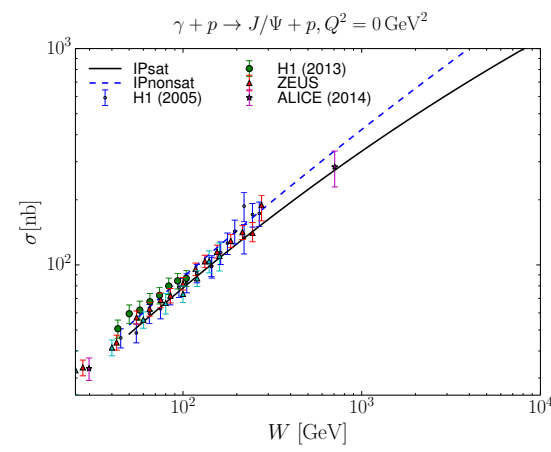

Fig. 1. Total cross section for $J / \Psi$ photoproduction as a function of collision energy from ${ }^{3}$.

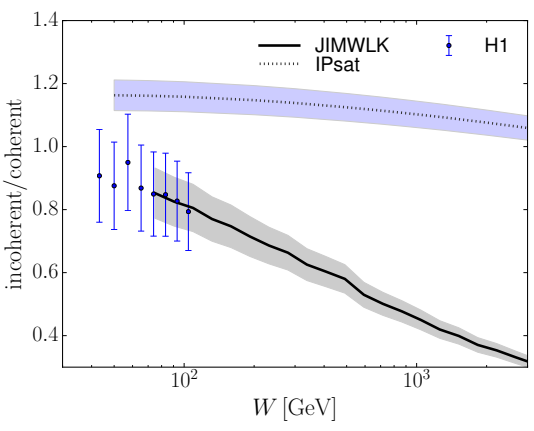

Fig. 2. Incoherent-to-coherent cross section ratio as a function of $W$ from $^{4}$

tude $N$ describes the scattering of a dipole with size roff the target, with the impact parameter $\mathbf{b}$. Here $T$ and $L$ refer to the photon polarization.

When the target remains intact, we talk about coherent diffraction and the cross section can be written as $\frac{\mathrm{d} \sigma^{\gamma^{*} p \rightarrow V p}}{\mathrm{~d} t}=\frac{1}{16 \pi}\left|\left\langle\mathcal{A}_{T, L}^{\gamma^{*} p \rightarrow V p}\right\rangle\right|^{2}$. Here the avarage \langle\rangle is taken over the possible configurations of the target, and consequently the cross section is sensitive on the average bdependence of the dipole-target interaction. On the other hand, if one subtracts the coherent contribution from the total diffractive cross section, the incoherent contribution where the target is required to break up remains: $\frac{\mathrm{d} \sigma^{\gamma^{*} p \rightarrow V p^{*}}}{\mathrm{~d} t}=\frac{1}{16 \pi}\left(\left\langle\left|\mathcal{A}^{\gamma^{*} p \rightarrow V p}\right|^{2}\right\rangle-\left|\left\langle\mathcal{A}^{\gamma^{*} p \rightarrow V p}\right\rangle\right|^{2}\right)$. As this cross section is a variance, it measures the amount of event-by-event fluctuations in the impact parameter dependence of $N$, and thus the amount of density fluctuations.

The Bjorken- $x$ evolution of the dipole amplitude $N$ can be computed perturbatively (see e.g. ${ }^{6}$ ) However, the initial condition for the evolution is non-perturbative and is usually obtained by fitting the HERA structure function data. When considering exclusive processes additional complications arise as one has to describe also the geometry evolution, which is sensitive to infrared dynamics. For example in Ref. ${ }^{4}$ the geometry evolution from JIMWLK evolution equation was included in the analysis of the HERA data. An alternative approach is to parametrize the $x$ dependence and geometry as e.g. in the IPsat model.

Different experiments at HERA and at the LHC have measured $J / \Psi$ production in photon-proton interaction at different center-of-mass energies $W$. As this cross section is generally expected to be sensitive to saturation effects, in Ref. ${ }^{3}$ the IPsat model parametrization for the dipole-proton interaction, and its linearized version (IPnonsat), were fitted to the HERA structure function data. In both cases, equally good description of the total cross section data was obtained. Then, the vector meson production as a function of $W$ was calculated and compared with the available data. The results are shown in Fig. 1, where it is seen that the non-linear effects 


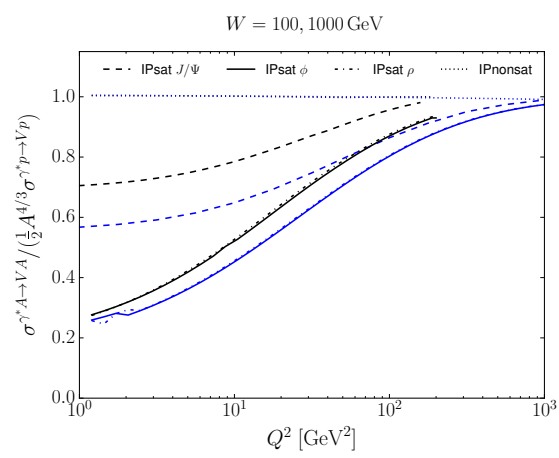

Fig. 3. Nuclear suppression factor for coherent vector meson production from ${ }^{3}$.

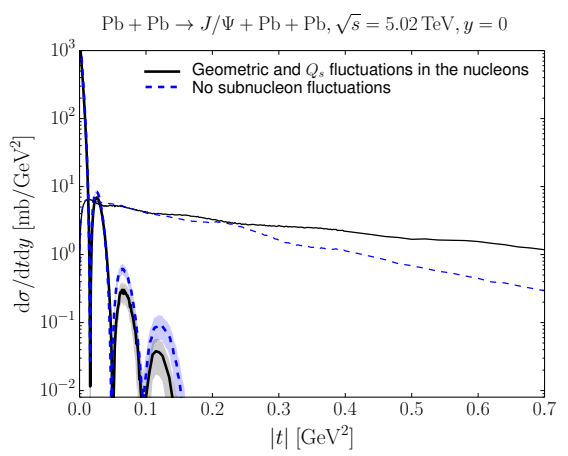

Fig. 4. $J / \Psi$ production cross section as a function of squared momentum transfer from ${ }^{9}$.

are small in the currently available energy range with proton targets.

The geometry evolution in Ref. ${ }^{4}$ makes it possible to study how the eventby-event fluctuations evolve towards small $x$. As discussed in Refs. ${ }^{7,8}$, the eventby-event fluctuating proton shape can be constrained by requiring a simultaneous description of the coherent and incoherent $J / \Psi$ production $t$ spectra from HERA. This analysis shows that at $x \approx 10^{-3}$ the fluctuations are significant. Then, one can perform a JIMWLK evolution down to small $x$. What is found in Ref. ${ }^{4}$ is that the evolution makes protons smoother at long distance scales. This causes the incoherent cross section to grow more slowly than the coherent cross section (note that in the black disk limit the incoherent cross section is suppressed as it gets contributions only from the edges of the target). This observation is compatible with the HERA measurements as shown in Fig. 2. In the IPsat model, on the other hand, there is no geometry evolution and the cross section ratio is constant.

\section{From protons to heavy nuclei}

As gluon densities at given transverse point are enhanced by roughly $A^{1 / 3}$, vector meson production off nuclei is a promising process to look for saturation effects ${ }^{10}$.

The size rof the interacting dipole is set by the vector meson mass $M_{V}$, such that $|\mathbf{r}| \sim 1 / M_{V}$. Very small dipoles have vanishing interaction probability due to the color transparency. Similarly, larger dipoles interact more strongly and as such are more sensitive to saturation effects. In Fig. 3 the cross section for exclusive vector meson production off gold nucleus is shown, divided by the same cross section off protons normalized such that in the absence of non-linear effects the ratio would be unity. This indeed is the case when the linear IPnonsat model is used to calculate dipole-nucleus interaction.

In the IPsat parametrization which includes saturation, the suppression factor is found to depend strongly on the meson mass, the light $\rho$ and $\phi$ mesons being more heavily suppressed than $J / \Psi$. In Fig. 3 the suppression is shown as a function of photon virtuality $Q^{2}$, which also controls the dipole size. At large virtualities the 
dipoles are in general smaller and suppression vanishes. However, this transition is relatively slow, due to the significant contribution from the large dipoles to the transverse cross section even at large $Q^{2}$, see Ref. ${ }^{3}$ for more details.

Finally in Fig. 4 the coherent and incoherent $J / \Psi$ photoproduction cross section off lead are shown in the LHC kinematics. The results are shown in two cases: with proton shape and normalization $\left(Q_{s}\right)$ fluctuations, and with nucleons having no substructure (dashed lines). As the substructure has little effect on average geometry, the coherent cross sections are compatible. The incoherent cross sections, on the other hand, are very different at $|t| \gtrsim 0.2 \mathrm{GeV}^{2}$, which corresponds to the distance scale of the hot spots in nucleons.

\section{Outlook}

New nuclear DIS data at high energies, both from the ultra peripheral heavy ion collisions at the LHC and from the future EIC, will provide us a new window for the studies of non-linear dynamics in the dense QCD matter. In addition to saturation effects, it will also be possible to study the evolution of the fluctuating transverse geometry, which is fundamentally interesting and provides crucial input for the modelling of Quark Gluon Plasma production.

\section{Acknowledgments}

This work was supported by the Academy of Finland, project 314764 , and by the European Research Council, Grant ERC-2015-CoG-681707.

\section{References}

1. M. Ryskin, Diffractive $J / \Psi$ electroproduction in LLA QCD, Z. Phys. C57, 89 (1993).

2. F. Gelis, E. Iancu, J. Jalilian-Marian and R. Venugopalan, The Color Glass Condensate, Ann. Rev. Nucl. Part. Sci. 60, 463 (2010).

3. H. Mäntysaari and P. Zurita, In depth analysis of the combined HERA data in the dipole models with and without saturation, Phys. Rev. D98, p. 036002 (2018).

4. H. Mäntysaari and B. Schenke, Confronting impact parameter dependent JIMWLK evolution with HERA data, Phys. Rev. D98, p. 034013 (2018).

5. H. Kowalski, L. Motyka and G. Watt, Exclusive diffractive processes at HERA within the dipole picture, Phys. Rev. D74, p. 074016 (2006).

6. I. Balitsky, Operator expansion for high-energy scattering, Nucl. Phys. B463, 99 (1996).

7. H. Mäntysaari and B. Schenke, Evidence of strong proton shape fluctuations from incoherent diffraction, Phys. Rev. Lett. 117, p. 052301 (2016).

8. H. Mäntysaari and B. Schenke, Revealing proton shape fluctuations with incoherent diffraction at high energy, Phys. Rev. D94, p. 034042 (2016).

9. H. Mäntysaari and B. Schenke, Probing subnucleon scale fluctuations in ultraperipheral heavy ion collisions, Phys. Lett. B772, 832 (2017).

10. H. Mäntysaari and R. Venugopalan, Systematics of strong nuclear amplification of gluon saturation from exclusive vector meson production in high energy electronnucleus collisions, Phys. Lett. B781, 664 (2018). 